Original Research Paper

\title{
Proposed Insights in the Pharmaco-Dynamic Theory of Ascorbate Pro-Oxidant Activity
}

\author{
Kheir Eddine Kerboua, Fatma Haiba, Fatima Bendjemia, \\ Mimouna Kerrache and Amina Boumediene \\ Unit of Immunology, Military University Hospital of Oran, HMRUO, Oran 31,000, Algeria
}

\author{
Article history \\ Received: 10-08-2015 \\ Revised: 23-08-2015 \\ Accepted: 03-11-2015 \\ Corresponding Author: \\ Kheir Eddine Kerboua \\ Unit of Immunology, Military \\ University Hospital of Oran, \\ Oran 31,000, Algeria
}

Mobile: +213541239163

Email: K.K.Eddine@gmail.com

\begin{abstract}
Ascorbate pro-drug theory promising possible effective and nontoxic treatment is intensively examined and centered on the importance of both extracellular and intra-cellular effects of ascorbate. In this study, we sought to determine the in vitro cell responsiveness in a plasma free milieu with or without an extracellular catalyst and/or hydrogen peroxide. This project brought to light how ascorbic acid would react to in a Red Blood Cell (RBC) suspension by using the two anti-oxidant pathways. We demonstrated that while ascorbate doesn't require an extracellular metal catalyst to induce cell damage via the lipid peroxidation pathway, the protein-centered metal pathway could be enhanced by a divalent cation, hydrogen peroxide or both. The current lack of understanding of ascorbate potential mechanism excites us to propose some insights for the vitamine $\mathrm{C}$ pharmacologic dose pro-oxidant activity.
\end{abstract}

Keywords: Ascorbic Acid, Pharmacodynamics, Pro-Oxidant, Biochemical, Mechanical and Enzymatic Biomarkers, Intracellular and Extracellular Effects, Human Erythrocyte

\section{Introduction}

Ascorbic Acid (AA, vitamin C, ascorbate) is well known for its antioxidant activity, but its proposed prooxidant activity at high concentrations that remains controversial (Wilson et al., 2014). To see whether ascorbate acts as a pro-oxidant, Suh et al. (2003) have conducted an experimental study using human plasma. They showed that even in the presence of redox-active iron or copper and hydrogen peroxide, ascorbate acts as an antioxidant to prevent lipid peroxidation and protein oxidation (Suh et al., 2003). However, Chen et al. (2007) has demonstrated in an animal model that $\mathrm{H}_{2} \mathrm{O}_{2}$ induced by Ascorbic Acid (AA) in presence of a divalent cation are strongly destroyed by the plasmatic defense although it still highly reactive in tissues (Chen et al., 2007). These two pilot studies have evoked more questions than answers and suggested that high doses of AA are prooxidant but without any damage effects on human plasma because of plasmatic $\mathrm{H}_{2} \mathrm{O}_{2}$ scavengers. Consequently, the Pro-Drug Theory of ascorbate was born. Nevertheless, it is clear that method and timing of evaluation of such new concepts is hindered by our limited mechanistic understanding and the lack of specific biomarkers (Chen et al., 2005). Needless to reminder that erythrocytes as like as neutrophils are considered the main system of AA recycling in human.
In this way, erythrocytes are more important because they have the particularity to be the first line of antioxidant defenses and are exposed to decreases of their antioxidant capacity (Chatterjee et al., 2008). Considering these points together, we have conducted an in vitro study to show the impact of the high doses of AA on human cells exempted from plasma milieu and to try to shed light onto the mechanistic sides.

\section{Research Design and Methods}

Ascorbic acid solutions: of 0 to $90 \mathrm{mM}, 20$ dilutions were prepared using a stock solution of $340 \mathrm{mM}$.

Human cells: we have chosen the Red Blood Cell (RBC) for its well defined protein-centered metal, Hemoglobin and other features resumed below (Fig. 1).

Preparation of erythrocytes suspension: Human blood from healthy donors, anti-coagulated with heparin, was stored at $4{ }^{\circ} \mathrm{C}$ and used for experiment in the same day. Human erythrocytes were isolated by centrifugation (10 $\mathrm{min}, \times 150 \mathrm{~g}$ ); plasma and buffy-coat removed and the cells washed three times with isotonic saline $(0.15 \mathrm{M} \mathrm{NaCl})$ at room temperature. Washed erythrocytes were suspended in saline to reach a concentration of about $3 \times 10^{5}$ cells $\mathrm{mL}^{-1}$. 


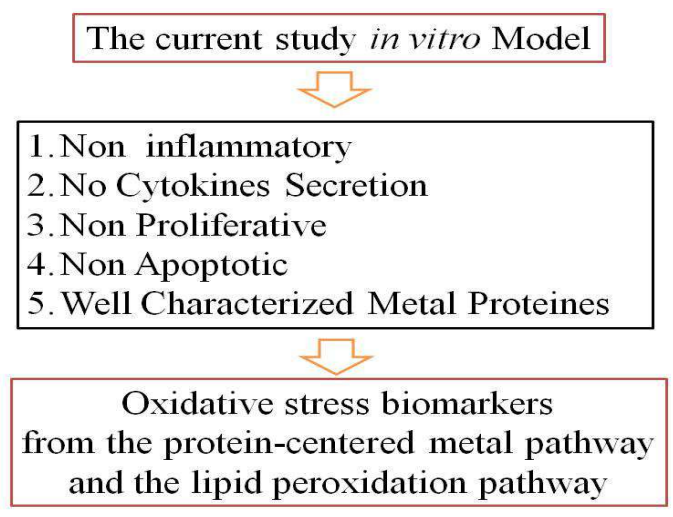

Fig. 1. Characteristics of human RBC as cell based model of in vitro studying oxidative stress responsiveness

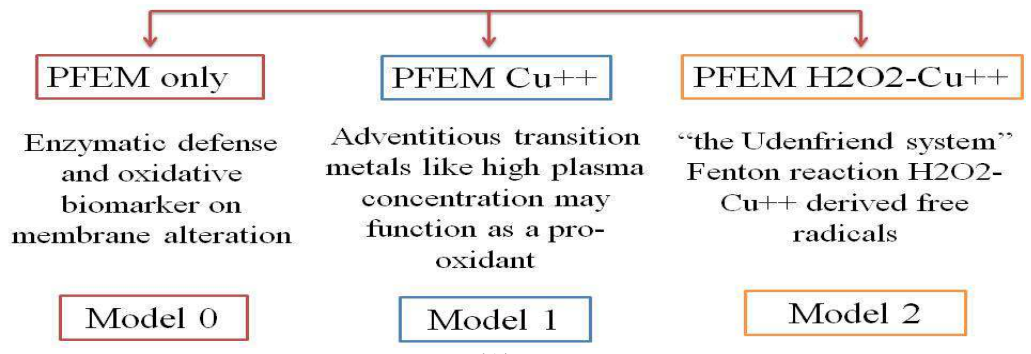

(A)

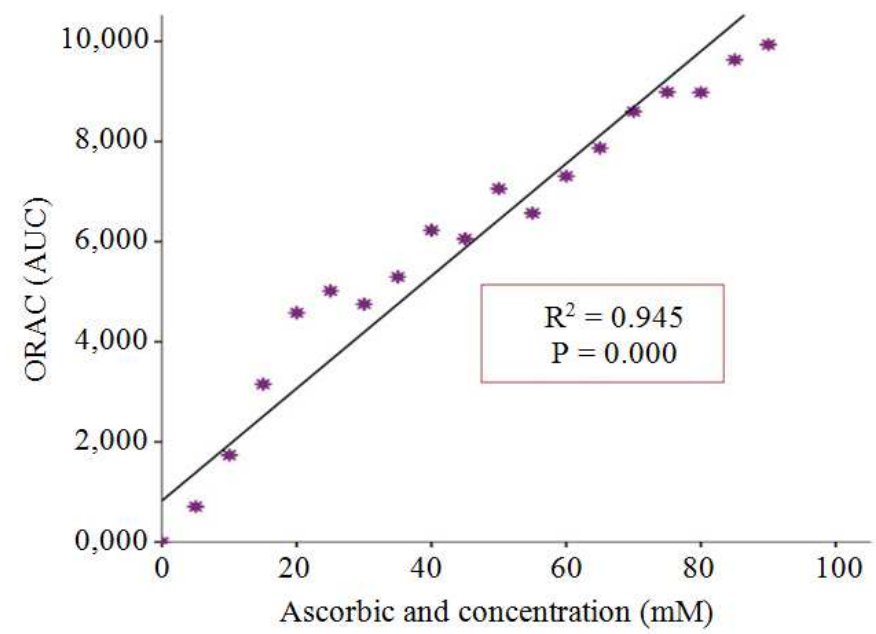

(B)

Fig. 2. The current study experimental conditions. (A) Characteristics of the three different cell suspension models. (B) ORAC value for each ascorbate solution showed that ascorbate dilutions keep their antioxidant capacity in solution. PFEM: Plasma Free Erythrocyte model. ORAC: the referential antioxidant assay; the Oxygen Reactive Absorbance Capacity

Three cell suspension models were used. Briefly, Model 0 contained Human RBC $(50 \mu \mathrm{L})+$ Solutions of Ascorbic acid $(50 \mu \mathrm{L})$; Model 1 consisting in incubation of human RBC $(50 \mu \mathrm{L})+$ Solutions of Ascorbic acid (50 $\mu \mathrm{L})+\mathrm{Cu}^{++} \mathrm{SO} 4(10 \mu \mathrm{L})$. Finally the model 2 contained human RBC $(50 \mu \mathrm{L})+$ Solutions of Ascorbic acid (50 $\mu \mathrm{L})+\mathrm{Cu}^{++} \mathrm{SO} 4(10 \mu \mathrm{L})+\mathrm{H}_{2} \mathrm{O}_{2} 10 \mathrm{~V}(25 \mu \mathrm{L})$ (Fig. $\left.2 \mathrm{~A}\right)$. The incubation was performed at $8^{\circ} \mathrm{C}$ during $18 \mathrm{~h}$.
Quantitative methods (Hopkins and Tudhope, 1974; Stephen and Low, 1985; Beutler, 1982; Marklund and Marklund, 1974; Glatzle et al., 1974; Dodge et al., 1963; Winterbourn, 1990): Mechanical, biochemical and enzymatic assessment were performed for each cell suspension. First of all, the total antioxidant capacity of different AA dilutions was assessed by the Oxygen Reactive Absorbance Capacity (ORAC) assay as 
described by Ou et al. (2002). The effects of the AA on the RBCs were assessed by counting the blit cells and osmotic fragility for the membrane deformability as described by Hopkins and Tudhope (1974); Malanodialdehyde and 4-HNE for the lipid peroxydation as described by Quintanilha et al. (1982) and Kinter (1996) respectively, Heinz bodies were measured by two assays; the colorometric assay of Winterbourn (1990) and the microscopic one of Stephen and Low (1985). Finally we have evaluated the activity of catalase, peroxydase and superoxide dismutase as described by Beutler (1982; Glatzle et al., 1974; Marklund and Marklund, 1974) respectively. RBC membrane was isolated according to the procedure of Dodge et al. (1963) The concentration of oxy-Hb and met-Hb were determined in by spectroscopy the haemolysate by measuring the absorbance at $\lambda_{577} \mathrm{~nm}$ and $\lambda_{630} \mathrm{~nm}$ as described by Winterbourn (1990) (OxyHb $[\mu \mathrm{M}]=66 \mathrm{~A}_{577}-80 \mathrm{~A}_{630}$; Met-Hb $\left.[\mu \mathrm{M}]=279 \mathrm{~A}_{630}-3 \mathrm{~A}_{577}\right)$.

Statistical analysis: Logistic regression, odds ratio, Mann-Whitney U test, Spearman correlations and graphics were performed using SPSS (IBM software version 20.0 Chicago-USA).

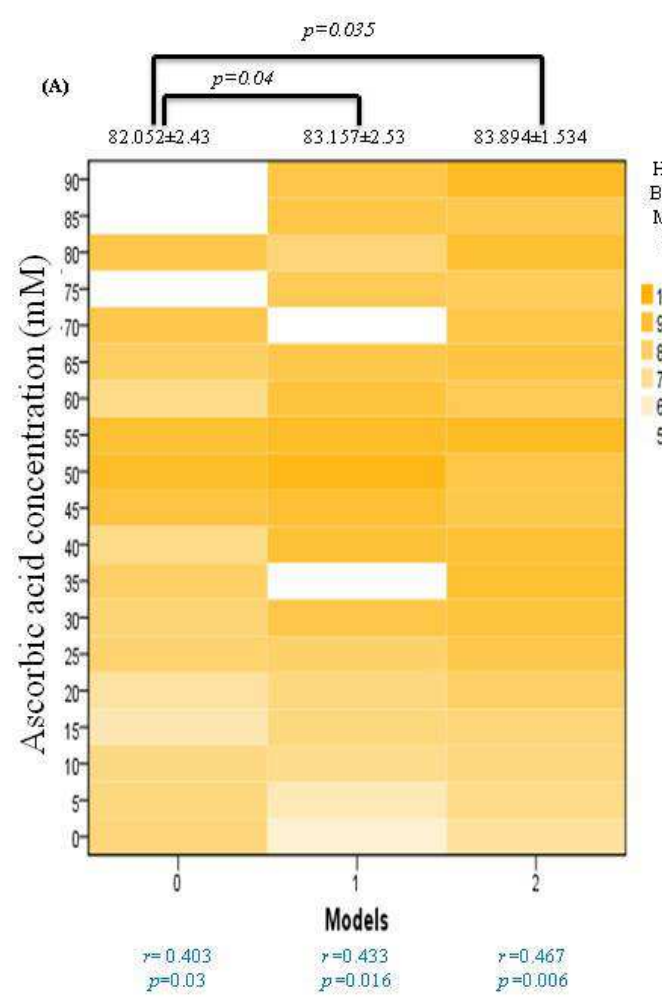

\section{Results}

We first investigated whether pharmacologic concentrations of ascorbate keep their antioxidant capacity in solution or not. Obviously, a positive correlation was obtained between the different concentrations of AA and their antioxidant capacity assessed by ORAC index (Fig. 2B). Then, RBCs oxidative responsiveness was studied in detail to determine the different impacts of AA on their antioxidant defense. The roles of intracellular versus extracellular catalyst in causing cell oxidative damage were examined, using three models: (i) model 0: AA alone that cannot induce $\mathrm{H}_{2} \mathrm{O}_{2}$ generation unless in intracellular compartment via a Fenton reaction with the proteincentered metal; (ii) model 1: Ascorbate with copper cations as a generator of $\mathrm{H}_{2} \mathrm{O}_{2}$; and finally (iii) model 2 which is an Udenfriend system. Data from the three models together show that ascorbate induces Heinz body formation in a dose dependant manner and the high levels of these aggregates are noticed only when ascorbate was present simultaneously with copper and $\mathrm{H}_{2} \mathrm{O}_{2}$ (Fig. 3A).
(B)

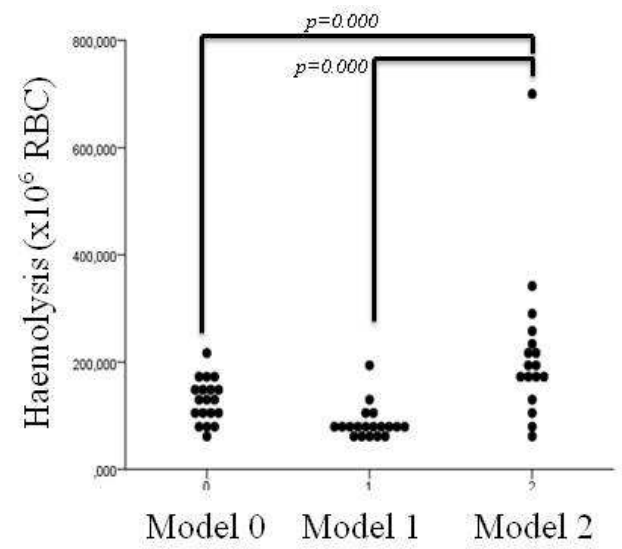

(C)

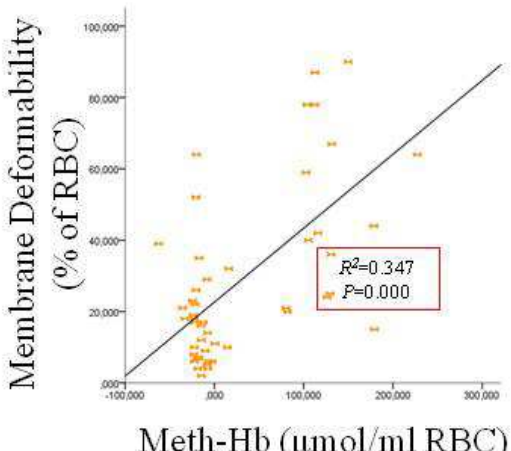

Fig.3. Mechanical cell damage following ascorbate exposure among the three RBC suspension models. (A) Hot-plot shows that Heinz bodies generation correlates positively with AA and figures out the comparison of Heinz bodies levels among the three cell suspension models. (B) Haemolysis among the three models shows that extracellular catalyst (copper ions in model 1) induces less impact in cell lysis than ascorbate alone. This graphic shows also that Udenfriend system (model 2) has the most heamolytic power. (C) Membrane deformability (assessed by percentage of blits cells) correlates significantly only with the earliest oxidative forms of the protein-centered metal (Met-Hb) 
Moreover, cell sensitivity to $\mathrm{AA}+\mathrm{H}_{2} \mathrm{O}_{2}$ exposure was greater by an increasing in cell damage, measured by RBC haemolysis (Fig. 4B). Interestingly, our statistical analysis highlights that the cell deformability was only associated with Met-hemoglobine (Fig. 3C).

Our approach to investigate whether intracellular antioxidant enzymes activities are associated with membrane impairment and lipid peroxidation reveals that the most involved enzyme in the antioxidant defense against ascorbate oxidative attack is the peroxydase which is associated with high level of
Malondialdehyde (MDA) (Fig. 5A). In a purpose to find an eventual link between the oxidative biochemical markers and the oxidative mechanical biomarkers, our data showed that cell osmotic fragility correlates significantly with MDA (Fig. 5C). Using the Receiver Operation Curves (ROC), an MDA threshold was obtained; when MDA increases up to it, AA may induce osmotic fragility (Fig. 5D). Concerning the peroxydase activity, its decreases was associated with a high level of MDA and a value less than $0.211 \mathrm{U} / \mathrm{ml}_{\mathrm{RBC}}$ may predict MDA generation (Fig. 5B).

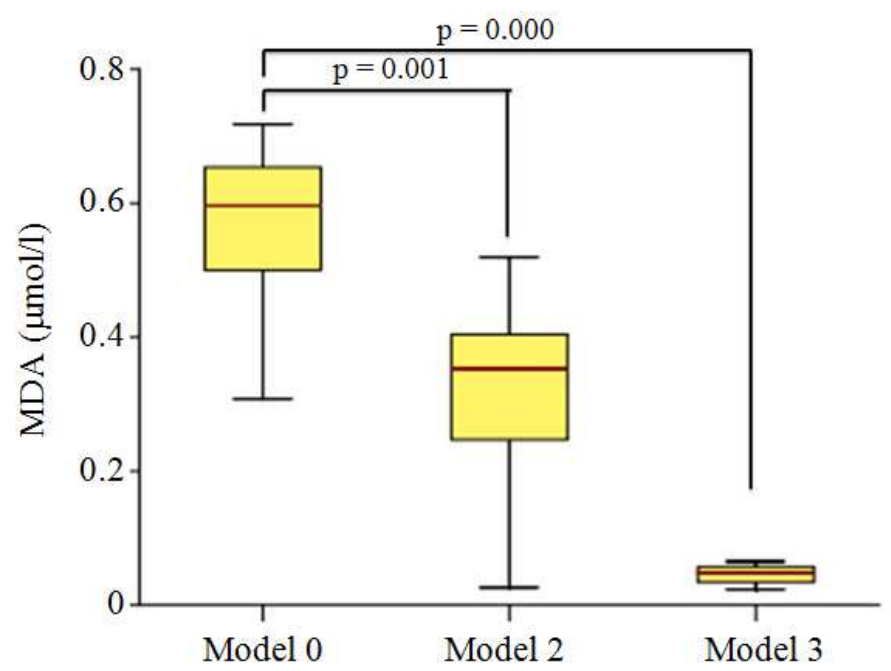

(A)
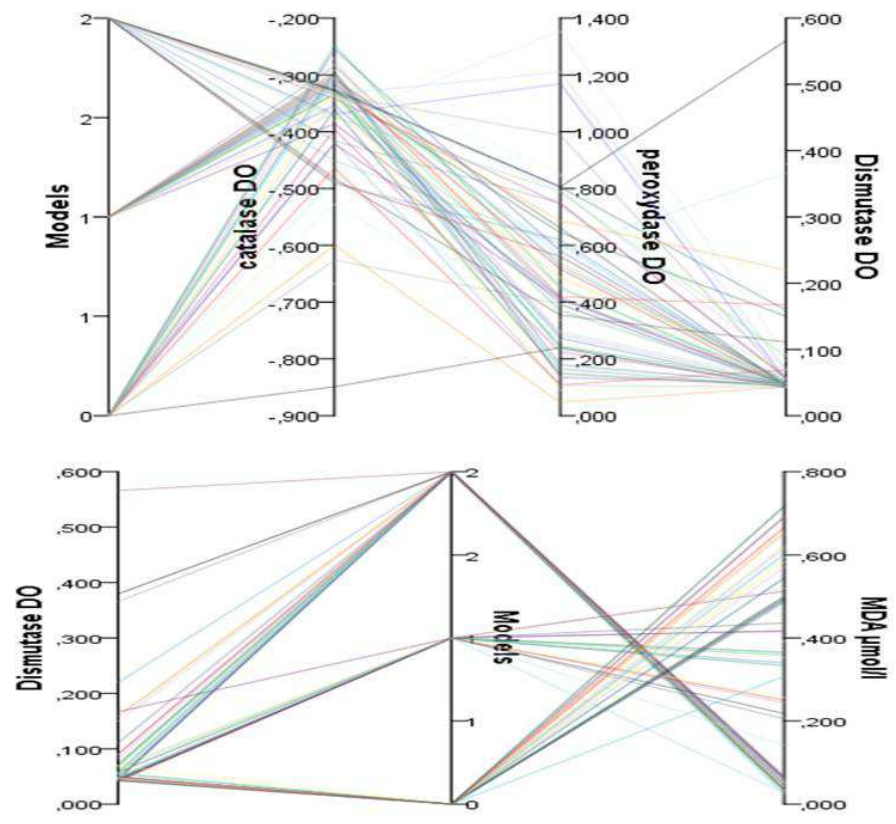

(B)

Fig. 4. Malondialdehyde formation under ascorbate oxidative attack (A) Comparison of MDA generation among the three models. (B) Parallel graphic shows the distrubution of MDA values and enzymatic activities accros the three cell suspension models 


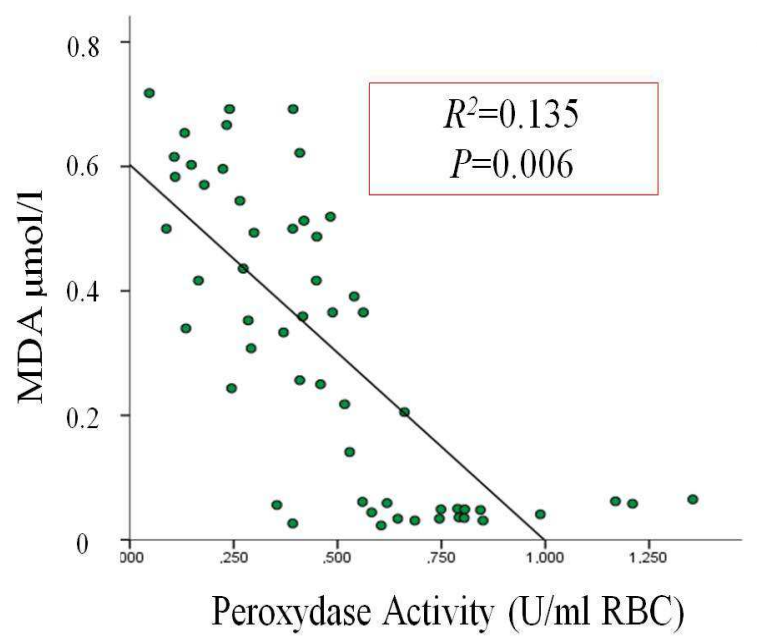

(A)

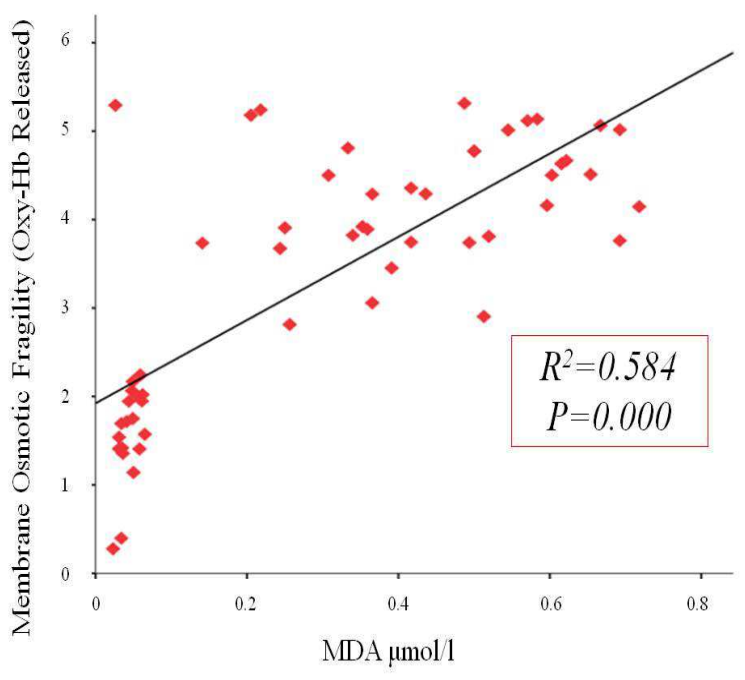

(C)

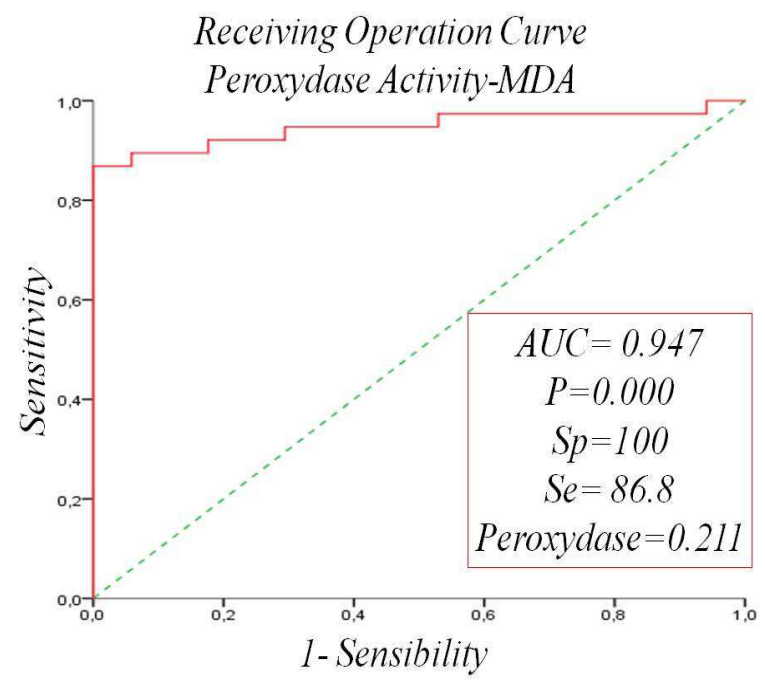

(B)

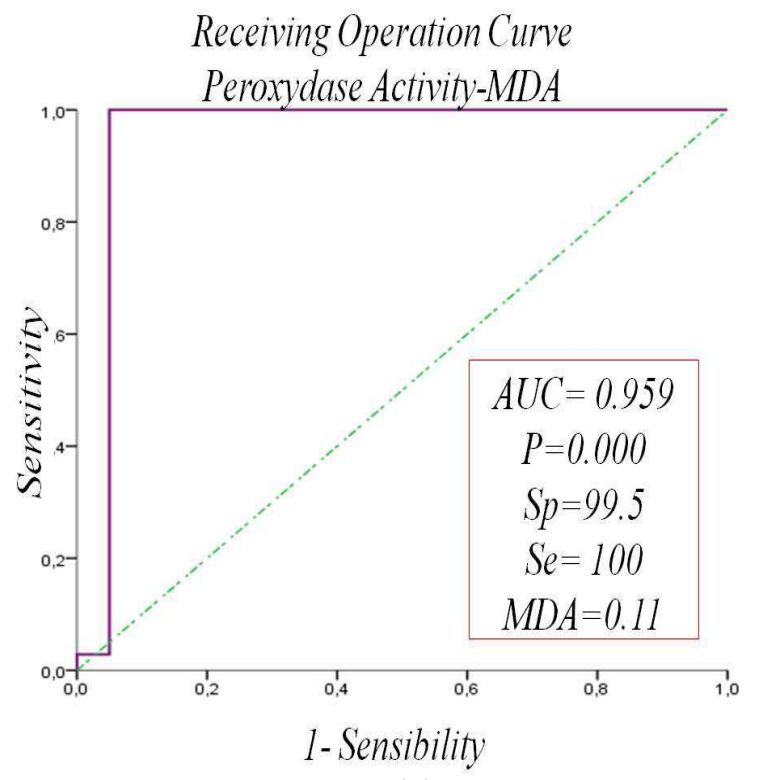

(D)

Fig.5. In vitro relationship between enzymatic defense, oxidative biochemical biomarker (MDA) and membrane fragility assessed by osmotic assay. MDA correlates with proxydase activity under a threshol of $0.211 \mathrm{U} / \mathrm{ml}_{\mathrm{RBC}}$. Graphic (A) shows a negative correlation between MDA and peroxydase activity while the grpagic (B) shows the peroxydase activity cut-off calculated by the Receiver Operation Curve. Graphic (C) shows a positive correlation between MDA and mambrane fragility. Graphic (D) shows the MDA cut-off up to it mechanical damage is induced

\section{Discussion}

It is known that cells respond to OS by two different pathways; the protein centred-metal pathway and the lipide peroxydation one (Goldberg and Stern, 1977; Trotta et al., 1981). OS induces transition of the RBC metallo-proteine (hemoglobine) from oxy- $\mathrm{Hb}_{\text {low spine }}$ to oxy-

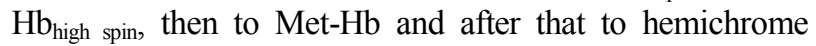
followed by intracellular aggregation of denatured hemoglobin called Heinz Bodies (HB) (Stephen and Low, 1985). The second pathway is the lipid peroxydation and depends mainly on the cell's enzymatic defense, essentially superoxyde dismutase and peroxidases. Theroritically, each element of these two pathways can be used as biomarker to assesse cell reponsivenesse to any molecule that may induce OS (Fig. 6).

Several arguments propose human $\mathrm{RBC}$ as a reliable model to study cell responsiveness to OS (Fig. 
1). Obviously, while RBCs are the most important system of AA recycling in the body, they contain the same biochemical and enzymatic defenses machinery that nucleic cells perform against free radicals. Moreover, their inability to release inflammatory mediators or to perform apotosis or proliferation makes them a well model for studying the prooxidant activity of ascorbyl radical by the evaluation of only the OS biomarkers. Furthermore, in our study, precautions were taken to minimize endogenous production of ROS by making cell's incubation at low temperature and empirically during $18 \mathrm{H}$. On other hand, the oxidative effect of ascorbate is proposed essentially as a safe medical alternative to treat cancer but only at high plasmatic doses which cannot be obtained except by intravenous infusion (IV) (Chen et al., 2008). Meanwhile, a large amount (mM) of ascorbate would be interacting with RBC. Basically, our data demonstrates the existence of a multitude of OS damage in $\mathrm{RBC}$ following incubation with $\mathrm{AA}$ at pharmacologic doses and that these effects occur in a dose dependant manner within the pharmacologic interval defined by Stephenson et al. (2013) (Fig. 3).

The current study highlights that ascorbate should be associated with $\mathrm{H}_{2} \mathrm{O}_{2}$ and copper cations in the extracellular milieu to induce cell damage by haemolysis (Fig. 3B).

However, we have discovered that this damage is not associated with lipid peroxidation (MDA) but with the first oxidative pathway that induces HB (Fig. 4). In this way, thanks to our models we confirm that $\mathrm{HB}$ is more involved in cell deformability than MDA. This latter is more involved in osmotic fragility (Fig. 5C and D). The present study explains the mystery noticed by Hopkins and Tudhope (1974) who has shown that there is no consistent relationship between mechanical fragility, glutathione peroxidase inhibition and HB formation (Hopkins and Tudhope (1974). The clinical relevance of these findings is that the pharmacologic doses of AA may involve heavily the first pathway of oxidative stress and make cells more sensitives to a physical attack like radiotherapy; this is of high interest in a cells with active metabolism like cancer cells (Fig. 6). This point is supported by several in vitro and in vivo data suggesting that vitamin $\mathrm{C}$ may overcome chemoresistance and improve chemosensitivity and cell fragility (Espey et al., 2011; Grad et al., 2001; Kurbacher et al., 1996; Song et al., 1995; Reddy et al., 2001). Besides, the presence of metal catalyst like copper ions should induce lipid peroxidation according to the pro-drug theory, but in our study we demonstrate that the highest levels of MDA are obtained with ascorbate alone without copper neither in the Udenfriend system. This confirms that extracellular presence of catalyst or $\mathrm{H}_{2} \mathrm{O}_{2}$ is not necessary for lipid peroxydation and, in contrary, may block it (Fig.5). Based on these data, the most cogent explanation of ascorbate action in inducing lipid peroxydation is by its intracellular mechanisms. Several data highlight that the most common theory of cytotoxic effect of high dose of AA is the intracellular and extracellular induction of $\mathrm{H}_{2} \mathrm{O}_{2}$ without demonstrating whether this will translate to a clinical benefit (Chen et al., 2007; 2005). Morever, despite the catalyst could not be identified; Deubzer et al. (2010) suggested that it could be a metallo-protein secreted by some tumor cells (Deubzer et al., 2010). Hereby, our data concord with this study's conclusions in the protein centered metal but not with the extracellular $\mathrm{Cu}^{++}$as a catalyst. Thus, we can hypothesize that this mechanism is more adapted to the intracellular theory than the extracellular one. The above discussion provides an insight into the role of metals capable of direct or indirect generation of free radicals through various mechanisms. Among these mechanisms, Fenton-and Haber-Weiss type reactions are most common and efficient into the formation of the superoxide and hydroxyl radicals (Fig. 6). On other hand, we have found that among the cell enzymatic defense, dismutase is more involved in OS and MDA generation, which is in agreement with several studies especially the work of Wilson et al. (2014). Furthermore, we confirme that peroxidase is also implicated in this process when its activity decreases under $0.211 \mathrm{U} / \mathrm{ml}_{\mathrm{RBC}}$ (Fig. 5A and B). Needless to say that the process of lipid peroxidation is catalyzed by iron and results in the formation of peroxyl radicals (ROO•). Once formed, peroxyl radicals can be rearranged via a cyclisation reaction to endoperoxides (precursors of malondialdehyde) with the final product of the peroxidation process being MDA (Fig. 6). Chen et al. (2008; 2007; 2005) suggested that ascorbate, at pharmacological concentrations, is a pro-drug for $\mathrm{H}_{2} \mathrm{O}_{2}$ delivery to tissues with major therapeutic implications. However, such conclusions may be criticized viewing the limits of the experimental model and don't concord with our data that showed that AA alone (model 0) has a low impact on the first oxidative pathway compared to $\mathrm{PFEM}+\mathrm{Cu}^{++}$and PFEM $+\mathrm{H}_{2} \mathrm{O}_{2}+\mathrm{Cu}^{++}$. Thereby, we found that $\mathrm{AA}$ is necessary but not sufficient for prooxidative damage based activity by this way and necessitates the presence of copper cations and $\mathrm{H}_{2} \mathrm{O}_{2}$. Of note, meeting these three elements $\left(\mathrm{AA}+\mathrm{Cu}^{++}+\mathrm{H}_{2} \mathrm{O}_{2}\right)$ allows a highest degree of haemolysis (Fig. 3B). Data from the three models indicate that the protein centered metal is associated with the cell deformability which correlates significantly with met-Hb. Although the lack of understanding of its potential mechanism, we propose new insights in the pharmacodynamic theory of prooxydant activity of Vitamine $\mathrm{C}$ on human cell. We concentrate essentially on the intracellular theory with focusing in the lipid peroxydation pathway (Fig. 6). 


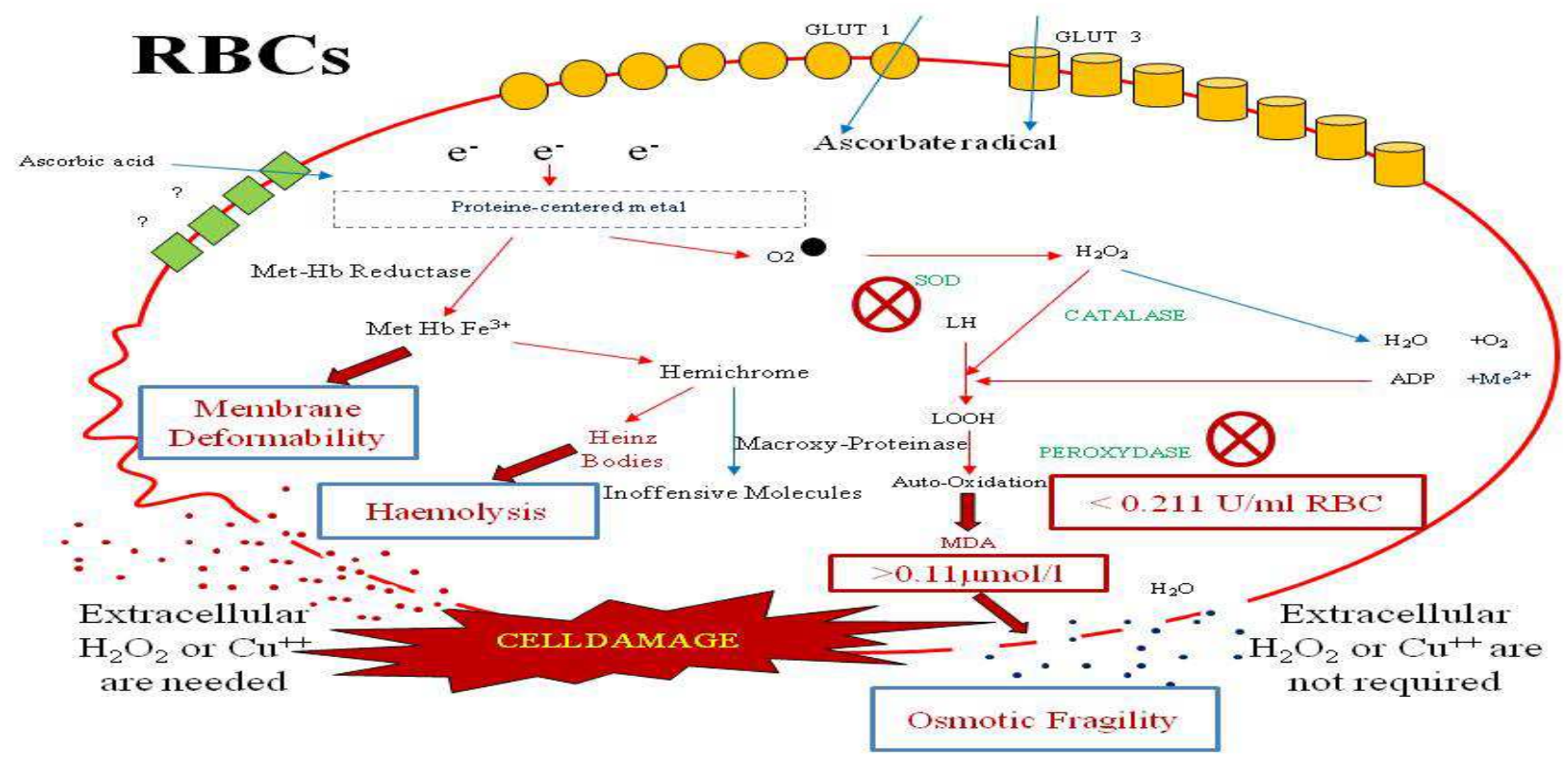

Fig.6. Proposed mechanistic insights of the two antioxidant pathways carried out by a cell under pharmacologic doses of ascorbate. While ascorbate enters to the cell by the glucose membrane transporters Glut 1 and 2, it faces two antioxidative pathways: the protein-centered metal one and the lipid peroxidation one. In the case of overwhelming these antioxidative resistance, toxic molecules will be generated and cause, among others, a multiple mechanical cell damage even in the absence of an extracellular catalyst or $\mathrm{H}_{2} \mathrm{O}_{2}$; Blue arrow: Physiological process, red arrow: Pathological process, decreased enzyme activity is shown by $\otimes$

Finally, beside the principal purpose of this study, many other applications may have to be considered. For instance, several studies argued that AA is still a good potential blood storage additive even it interacts with oxy-Hb and produces met-Hb (Raval et al., 2010). According to our findings, such conclusions lack in accuracy because these studies usually use a limited mechanical and fragility assays. We state that blood storage complications are most likely caused by the progressive of both structural and functional changes in RBCs. Besides, another application field is the in vitro modeling of OS damage. In contrary to Chen et al. (2007) who argued that the immunodeficient mice with a xenograft is the most characterized method of predicting potential activity of a given molecule in humans, we propose a simple cellbased in vitro model (Chen et al., 2005). In fact, Chen's in vivo model induces a high level of ROS after hypoxic ischemia and cold ischemia of the xenograft and stimulates endothelial cell which might up-regulate AA transporters. Finally, in addition to the enzymatic defenses defects against OS in cancer cell, we should remind that cancer cells have the particularity to express high amount of AA receptors allowing ascorbate accumulation and therefore a highly specific oxidative damage. In this way we have to consider our findings about cell mechanical changes like membrane deformability and osmotic fragility instead of the biochemical changes and the end products of lipid peroxidation like MDA that have been reported to mediate cell toxicity and apoptosis in cancer therapy.

\section{Conclusion}

Emerging knowledge suggests that the role of ascorbate in cancer treatment should be re-examined, but as far as we know the unique pharmacodynamic explanation is based on its pro-oxidant damage. Therefore, the evidence falls into two categories: Clinical data on dose concentration relationships and laboratory data describing potential cell toxicity at high concentrations of ascorbate in cell lines. The current study used a human cell based in vitro model to try to figure out some elements about the mechanistic cytotoxicity that include an accelerated pro-oxidant damage via the cell's mechanical and biochemical impairment in a doseresponse manner. In the light of the recent discoveries like the evidence that MDA induces apoptotic signal, studies of the ascorbate pro-drug theory in a nucleic cell based in vitro model are required to improve our translational knowledge which is critical for future research.

\section{Acknowledgment}

The authors thank Professor Bey Baba-Hamed $\mathrm{PhD}$. for providing gift chemicals for MDA 
assessment. We wish to thank Professor Mourad Aribi, $\mathrm{PhD}$ for valuable discussions.

\section{Funding Information}

This study was supported by the Scientific Council of the Military University Hospital of Oran HMRUO. No. 2014/0003/01 and by the Unit of Innunology of HMRUO. Oran, Algeria.

\section{Author's Contributions}

Kheir Eddine Kerboua: Developed the ex-vivo model of the prooxidant theory of ascorbic acid, Coordinated the study, conducted oxidative stress analyses, integrated all the data and wrote the manuscript.

Fatma Haiba: Conducted data analysis and contributed to the manuscript preparation.

Fatima Bendjemia and Mimouna Kerrache: Conducted the big part of laboratory assessment of oxidative stress balance parameters.

Amina Boumediene: Contributed to the technical work and data analysis.

\section{Ethics}

The authors declare that there is no conflict of interest.

\section{References}

Beutler, E., 1982. Catalase. In: Red cell Metabolism, a Manual of Biochemical Methods, Beutler, E. (Ed.), Grune and Stratton, New York, pp: 105-116.

Chatterjee, M., R. Saluja, V. Kumar, A. Jyoti and G.K. Jain et al., 2008. Ascorbate sustains Neutrophil NOS expression, catalysis and oxidative burst. Free Radical Biol. Med., 45: 1084-1093.

DOI: 10.1016/j.freeradbiomed.2008.06.028

Chen, Q., M.G. Espey, M.C. Krishna, J.B. Mitchell and C.P. Corpe et al., 2005. Pharmacologic ascorbic acid concentrations selectively kill cancer cells: Action as a pro-drug to deliver hydrogen peroxide to tissues. Proc. Nat. Acad. Sci. USA, 102: 13604-13609. DOI: 10.1073 /pnas.0506390102

Chen, Q., M.G. Espey, A.Y. Sun, J.H. Lee and M.C. Krishna et al., 2007. Ascorbate in pharmacologic concentrations selectively generates ascorbate radical and hydrogen peroxide in extracellular fluid in vivo. Proc. Nat. Acad. Sci. USA, 104: 8749-8754. DOI: $10.1073 /$ pnas.0702854104

Chen, Q., M.G. Espey, A.Y. Sun, C. Pooput and K.L. Kirk et al., 2008. Pharmacologic doses of ascorbate act as a prooxidant and decrease growth of aggressive tumor xenografts in mice. Proc. Nat. Acad. Sci. USA, 105: 11105-11109.

DOI: $10.1073 /$ pnas.0804226105
Deubzer, B., F. Mayer, Z. Kuci, M. Niewisch and G. Merkel et al., 2010. $\mathrm{H}_{2} \mathrm{O}_{2}$-mediated cytotoxicity of pharmacologic ascorbate concentrations to neuroblastoma cells: Potential role of lactate and ferritin. Cell. Physiol. Biochem., 25: 767-774. DOI: $10.1159 / 000315098$

Dodge, J.T., C. Mitchell and D.J. Hanahan, 1963. The preparation and chemical characteristics of hemoglobin-free ghosts of human erythrocytes. Arch. Biochem. Biophys., 100: 119-130. DOI: 10.1016/0003-9861(63)90042-0

Espey, M.G., P. Chen, B. Chalmers, J. Drisko and A.Y. Sun et al., 2011. Pharmacologic ascorbate synergizes with gemcitabine in preclinical models of pancreatic cancer. Free Radical Biol. Med., 50: 1610-1619. DOI: 10.1016/j.freeradbiomed.2011.03.007

Glatzle, D., J.P. Vuilleumier, F. Webe and K. Decker, 1974. Glutathione reductase test with whole blood, a convenient procedure for the assessment of the riboflavin status in humans. Experientia, 30: 665-667. DOI: 10.1007/BF01921531

Goldberg, B. and A. Stern, 1977. The role of the superoxide anion as a toxic species in the erythrocyte. Arch. Biochem. Biophys., 178: 218-225. DOI: 10.1016/0003-9861(77)90187-4

Grad, J.M., N.J. Bahlis, I. Reis, M.M. Oshiro and W.S. Dalton et al., 2001. Ascorbic acid enhances arsenic trioxide-induced cytotoxicity in multiple myeloma cells. Blood, 98: 805-813. DOI: 10.1182/blood.V98.3.805

Hopkins, J. and G.R. Tudhope, 1974. The effects of drugs on erythrocytes in vitro: Heinz body formation, glutathione peroxidase inhibition and changes in mechanical fragility. Br. J. Clin. Pharmacol., 1: 191-195. PMID: 22454946

Kinter, M., 1996. Free Radicals: A Practical Approach. 1st Edn., IRL Press at Oxford University Press, Oxford, ISBN-10: 0199635609, pp: 310.

Kurbacher, C.M., U. Wagner, B. Kolster, P.E. Andreotti and D. Krebs et al., 1996. Ascorbic acid (vitamin C) improves the antineoplastic activity of doxorubicin, cisplatin and paclitaxel in human breast carcinoma cells in vitro. Cancer Lett., 103: 183-189. DOI: 10.1016/0304-3835(96)04212-7

Marklund, S. and G. Marklund, 1974. Involvement of the superoxide anion radical in the autoxidation of pyrogallol and a convenient assay for superoxide dismutase. Eur. J. Bio-chem., 47: 469-474. DOI: $10.1111 /$ j.1432-1033.1974.tb03714.x

Quintanilha, A.T., L. Packer, J.M. Davies, T.L. Racanelli and K.J. Davies, 1982. Membrane effects of vitamin E deficiency: Bioenergetic and surface charge density studies of skeletal muscle and liver mitochondria. Ann. N Y Acad. Sci., 393: 32-47. PMID: 6959560 
Raval, J.S., J.H. Waters, A. Seltsam, E.A. Scharberg and E. Richter et al., 2010. The use of the mechanical fragility test in evaluating sublethal RBC injury during storage. Vox Sanguinis, 99: 325-331. DOI: $10.1111 /$ j.1423-0410.2010.01365.x

Reddy, V.G., N. Khanna and N. Singh, 2001. Vitamin $\mathrm{C}$ augments chemotherapeutic response of cervical carcinoma HeLa cells by stabilizing P53. Biochem. Biophys. Res. Commun., 282: 409-415. DOI: $10.1006 /$ bbrc.2001.4593

Song, E.J., V.C. Yang, C.D. Chiang and C.C. Chao, 1995. Potentiation of growth inhibition due to vincristine by ascorbic acid in a resistant human non-small cell lung cancer cell line. Eur. J. Pharmacol., 292: 119-125. PMID: 7720783

Stephen, M.W. and P.S. Low, 1985. Hemichrome binding to band 3: Nucleation of Heinz bodies on the erythrocyte membrane. Biochemistry, 24: 34-39. DOI: $10.1021 / \mathrm{bi00322a006}$

Ou, B., M. Hampsch-Woodill, J. Flanagan, E.K. Deemer and R.L. Prior et al., 2002. Novel fluorometric assay for hydroxyl radical prevention capacity using fluorescein as the probe. J. Agric. Food Chem., 50: 2772-2777.

DOI: $10.1021 / \mathrm{jf} 011480 \mathrm{w}$
Stephenson, C.M., R.D. Levin, T. Spector and C.G. Lis, 2013. Phase I clinical trial to evaluate the safety, tolerability and pharmacokinetics of high-dose intravenous ascorbic acid in patients with advanced cancer. Cancer Chemotherapy Pharmacol., 72: 139-146. DOI: 10.1007/s00280-013-2179-9

Suh, J., B.Z. Zhu and B. Frei, 2003. Ascorbate does not act as a pro-oxidant towards lipids and proteins in human plasma exposed to redox-active transition metal ions and hydrogen peroxide. Free Radical Biol. Med., 34: 1306-1314.

DOI: $10.1016 / \mathrm{S} 0891-5849(03) 00147-3$

Trotta, R.J., S.G. Sullivan and A. Stern, 1981. Lipid peroxidation and hemoglobin degradation in red blood cells exposed to $t$-butyl hydroperoxide: Dependence on glucose metabolism and hemoglobin status. Biochim. Biophys. Acta (BBA)-General Subjects, 678: 230-237. DOI: 10.1016/0304-4165(81)90211-7

Wilson, M.K., B.C. Baguley, C. Wall, M.B. Jameson and M.P. Findlay, 2014. Review of high-dose intravenous vitamin $\mathrm{C}$ as an anticancer agent. Asia-Pacific J. Clin. Oncol., 10: 22-37. DOI: 10.1111/ajco.12173

Winterbourn, C.C., 1990. Oxidative reactions of hemoglobin. Methods Enzymol., 186: 265-272. DOI: $10.1016 / 0076-6879(90) 86118-F$ 